\title{
A importância da higiene oral em pacientes com ventilação mecânica
}

\section{The importance of oral hygiene in patients with mechanical ventilation}

\author{
Thaís Maria de Jesus' $\bullet$ Gabriela Ferreira Pimenta ${ }^{2} \bullet$ Luanna Pereira de Oliveira ${ }^{3}$ \\ Nathalia Christine Scott Marins ${ }^{4} \bullet$ Micheli Teixeira de Oliveira ${ }^{5}$
}

\begin{abstract}
RESUMO
A importância da higiene oral em pacientes com ventilação mecânica foi a temática do estudo, tendo como linha de pesquisa, a educação em enfermagem e área predominante, a enfermagem no cuidado ao cliente crítico adulto/ emergência. O objeto do estudo foi o enfermeiro e o objetivo era identificar a necessidade de aprimoramento dos profissionais de enfermagem na importância da higiene oral diária na terapia intensiva. Metodologia de abordagem bibliográfica, descritiva e qualitativa. Foi realizada uma pesquisa na biblioteca virtual em saúde, nas bases MEDLINE, LILACS e BDENF, utilizando os descritores: Equipe de enfermagem, Unidade de terapia intensiva e Cuidados de enfermagem. Após a busca, teve como resultado o quantitativo de dez artigos, onde foi realizada a leitura na íntegra. $O$ desenvolvimento e $\circ$ ideal do trabalho foram voltados para a melhoria na assistência da enfermagem ao paciente, o foco maior do trabalho está nos pacientes impossibilitados de exercer esse autocuidado, garantindo conforto e evitar complicações, afim de minimizar o tempo de internação. $O$ risco de infecções em um ambiente hospitalar existe, porém, cabe ao profissional atentar-se aos cuidados e manuseio do paciente. A importância da equipe multidisciplinar faz toda a diferença no que diz a respeito do cuidado, traçando metas e diretrizes eficazes a assistência.
\end{abstract}

Descritores: Equipe de enfermagem, Unidade de terapia intensiva e Cuidados de enfermagem

\begin{abstract}
The importance of oral hygiene in patients with mechanical ventilation was the subject of the study, having research education in nursing and nursing care predominantly critical customer adult/emergency. The object of study was the nurse and the goal was to identify the need for improvement of nursing professionals the importance of daily oral hygiene in intensive therapy.Methodology of bibliographic, descriptive and qualitative approach.A research on the virtual health library in the bases MEDLINE, LILACS and BDENF, using the key words: nursing, intensive care and nursing care.After the search, has resulted in the amount of ten articles, where the reading in full. The development and the ideal of work were aimed at improving nursing care to the patient, the increased focus of work is in patients unable to exercise that self-care, ensuring comfort and avoid complications, in order to minimize the length of hospitalization. The risk of infections in a hospital environment exists, however, it is up to the professional trying to care and patient handling. The importance of multidisciplinary team makes all the difference in the respect of care, tracing goals and guidelines effective assistance.
\end{abstract}

Keywords: Nursing Team, Intensive Care and Nursing Care.

'Pós-graduada na modalidade de residência em Enfermagem no Programa de Clínica Médica e Cirúrgica pela Universidade Federal do Estado do Rio de Janeiro (Unirio) Pós-graduada em auditoria em saúde pelo Instituto Brasileiro de Formação. Pós-graduada em enfermagem estética pelo Nepuga. Enfermeira contratada pelo Grupo ProntoBaby atuando no centro pediátrico da Lagoa. E-mail: thaismaria6@gmail.com. Autor correspondente.

${ }_{2}^{2}$ Pós-graduada na modalidade de residência em Enfermagem no Programa de Clínica Médica e Cirúrgica pela Universidade Estadual do Rio de Janeiro. Pósgraduada em terapia intensiva pala Universidade Cândido Mendes. Enfermeira contratada pelo Ministério da Saúde atuando no Hospital Federal de Bonsucesso.E-mail: gabriela.fpimenta@hotmail.com

${ }^{3}$ Pós-graduada na modalidade de residência em Enfermagem no Programa de Clínica Médica e Cirúrgica pela Universidade Federal do Estado do Rio de Janeiro. Pós-graduada no curso de especialização em Enfermagem oncológica pala Universidade Cândido Mendes. Enfermeira contratada pelo Ministério da Saúde atuando no Hospital Federal Cardoso Fontes. Enfermeira contratada pelo Ministério da Saúde atuando no Hospital Universitário Pedro ErnestoE-mail: luanninhah.rj@gmail.com

${ }^{4}$ Pós-graduada na modalidade de residência em Enfermagem no Programa de Clínica Médica e Cirúrgica pela Universidade Federal do Estado do Rio de Janeiro. Enfermeira contratada pelo Ministério da Saúde atuando no Hospital Federal de Bonsucesso. Enfermeira contratada pela Viva Rio atuando na Unidade de Pronto atendimento de Irajá.E-mail: scott.nathalia@yahoo.com.br

${ }^{5}$ Pós-graduada na modalidade de residência em Enfermagem no Programa de Clínica Médica e Cirúrgica pela Universidade Federal do Estado do Rio de Janeiro. Pós-graduada no curso de especialização em Enfermagem oncológica pala Universidade Cândido Mendes. Pós-graduanda no curso de Cardiologia e Hemodinâmica pela Faculdade Israelita de Ciências e Saúde Albert Einstein. Enfermeira contratada pela United Health Group-Amil.E-mail:michelitoliveira@gmail.com 


\section{INTRODUÇÃO}

A higiene oral é uma das condições básicas para a manutenção da saúde e conforto dos pacientes, pois as patologias que acometem dentes e gengivas podem levar a perdas de elementos, demais alterações na digestão dos alimentos, o que propiciam o surgimento de infecções bacterianas. A higiene oral consiste na conservação e na limpeza da cavidade bucal com objetivo da prevenção de cáries dentárias e de infecções bucais, digestivas e respiratórias'.

O cuidado da saúde oral é uma intervenção de enfermagem essencial. Para que a técnica de higiene oral resulte numa remoção eficaz dos restos alimentares e da placa bacteriana, seu objetivo é prevenir não somente as alterações na cavidade oral como evitar que esses microrganismos se tornem agentes complicadores do estado geral do paciente ${ }^{2}$.

A unidade de terapia intensiva (UTI) é um ambiente hospitalar onde ficam pacientes em estado crítico e normalmente apresentam uma condição deficiente da higiene oral. A proliferação das bactérias pode tornar responsável por complicações que elevam a taxa de mortalidade nestes pacientes ${ }^{3}$.

Como tema a atuação da enfermagem na saúde oral em pacientes na terapia intensiva, tendo área predominante de cuidado ao cliente crítico adulto/emergência está susceptivel a adquirir infecções no ambiente hospitalar. Sendo assim como linha de pesquisa a educação em enfermagem, que envolve um melhor entendimento da equipe sobre as práticas diárias de higiene oral, compete cumplicidade e comprometimento de toda equipe para um resultado satisfatório, e provocar uma reflexão nas condutas dos profissionais envolvidos na assistência.

$O$ objeto do estudo foi o enfermeiro relacionado às atividades da saúde oral do paciente na unidade de terapia intensiva. $O$ enfermeiro supervisiona a equipe de enfermagem, onde a mesma tem como uma de suas atribuições o cuidado com a higiene oral do paciente e ainda compete ao enfermeiro o planejamento, organização, coordenação, execução e avaliação dos serviços de assistência dos pacientes graves com risco de vida ${ }^{4}$.

Ter conhecimento teórico e prático com o cuidado da saúde oral, que vai além de conforto e devem ser adotadas técnicas e produtos, o qual deve ser compartiIhado com toda equipe de enfermagem para que o cuidado com a cavidade oral tenha sua importância associada também à prevenção de infecções. Os enfermeiros devem elaborar protocolos que possam ser exequíveis e promoverem treinamentos para as demais categorias de enfermagem ${ }^{5}$.

Como questionamento: $A$ equipe de enfermagem busca mecanismos e conhecimento sobre a importância da higiene oral para o conforto e bem-estar do paciente visando assim evitar complicações?
O objetivo do estudo foi identificar a necessidade de aprimoramento dos profissionais de enfermagem agregando e enfatizando a importância da higiene oral diária na terapia intensiva.

"A higienização deficiente da cavidade oral é o fator que favorece as infecções locais e serve de porta de entrada das infecções sistêmicas, comprometendo o estado geral do paciente e aumentando seu tempo de internação hospitalar".

"É de extrema importância que pacientes internados em UTI recebam cuidados de higiene oral suficiente durante sua permanência na unidade para evitar a manifestação ou complicação de patologias associadas à saúde bucal"’.

O conforto e a higiene oral são primordiais na recuperação do paciente pois ajudam na prevenção de possíveis doenças que podem ser desencadeadas com a ineficiência da higiene, portanto é de grande relevância a educação da sociedade sobre este assunto, desde a internação até a alta do paciente.

Portanto é primordial alertar aos profissionais da enfermagem os benefícios que a higiene oral traz ao paciente, tais como: qualidade de vida, promoção, prevenção de patologias e uma boa recuperação.A higiene oral diária é eficaz no processo saúde/doença, sendo relevante os debates e questionamentos, que visa melhorias, crescimento e valorização de todos os cuidados pelo profissional de saúde.

\section{METODOLOGIA}

O estudo evidencia a importância da higiene oral como meio de prevenir patologias diversas, realizar o controle de infecção da cavidade oral a fim de reduzir a mortalidade dos pacientes e o período de internação. $A$ importância está em conhecer a prática da higiene oral entre a equipe de enfermagem visando melhorar a qualidade da assistência e o conhecimento dos profissionais da equipe.

O estudo é de abordagem bibliográfica, descritivo com caráter fundamental em um estudo qualitativo, pois é por meio dele que os dados são coletados.

A pesquisa bibliográfica procura explicar um problema a partir de referências teóricas publicadas em artigos, livros, dissertações e teses. Pode ser realizada independentemente ou como parte da pesquisa descritiva ou experimental. É importante descrever com detalhes o passo a passo do experimento para que outros colegas vejam exatamente como o pesquisador chegou ás conclusões. $A$ ideia é permitir que outros possam repetir o experimento percorrendo o mesmo caminho. Dessa forma, a comunidade cientifica pode ver se a conclusão da pesquisa é replicável, isto é, se a mesma resposta é obtida quando mantidas as mesmas circunstâncias e repetidos os mesmos procedimentos ${ }^{8,9}$. 
Em princípio a descrição constitui a habilidade de fazer com que o outro veja mentalmente aquilo que o pesquisador observou. Em outras palavras, a descrição deve ser suficientemente precisa para que o interlocutor ou leitor seja capaz de visualizar exatamente aquilo que o pesquisador observou. A descrição se presta ainda para descrever metodologicamente cada um dos passos dados na realização da pesquisa e na aplicação das técnicas de pesquisa ${ }^{8}$.

Utilizamos a pesquisa qualitativa quando queremos descrever nosso objeto de estudo com mais profundidade. Por isso é muito comum em estudos sobre o comportamento de um indivíduo ou de um grupo social?.

Foi realizada uma pesquisa na biblioteca virtual em saúde nas bases, Medline, Lilacs e Bdenf, no mês de abril de 20I5, utilizando os descritores: Equipe de enfermagem, unidade de terapia intensiva e cuidados de enfermagem.

De forma inicial, após pesquisa dos descritores separadamente obteve-se os seguintes números:

Devido à grande quantidade de artigos encontrados, foi realizado um refinamento inicial, utilizando os descritores em dupla e estão apresentados na tabela 2:

Com um grande quantitativo de artigos encontrados, foi realizado um novo refinamento com os descritores em trio e estão apresentados na tabela 3 :

Através da leitura do resumo dos artigos encontrados na base de dados, descartando os de outra língua, foram selecionados os artigos que atendem ao objetivo do trabalho e estão apresentados na tabela 4.

\section{RESULTADOS E DISCUSSÃO}

A importância da higiene oral em pacientes com ventilação mecânica foi o tema abordado e embasado na deficiência dos procedimentos de higiene oral realizados pelos profissionais de saúde visando proporcionar ao paciente a higiene oral adequada evitando assim complicações secundárias.

O objetivo foi aprimorar o conhecimento das técnicas adequadas para realização da higiene oral. $O$ enfermeiro como educador tem o dever de buscar mecanismos de conhecimento teórico e prático a fim de manter uma educação continuada dentro de sua equipe visando a qualidade da assistência.

Para atender o objetivo foi realizada uma busca na biblioteca virtual em saúde, onde foram selecionados 10 artigos que estão apresentados na tabela 4 na metodologia.Após a seleção dos artigos, foi realizada a leitura na integra e agrupadas as ideias principais destes autores, que permitiu a divisão em 3 categorias: Cuidados de higiene oral, complicações decorrentes da falta de higiene oral e educação em enfermagem.

\section{Categoria I - Cuidados de higiene oral.}

O enfermeiro além de lidar com as atividades administrativas de supervisão e treinamento da equipe é preciso também atentar a mecanismos de prevenção a patologias decorrentes da higiene oral ineficaz. Estabelecendo metas diárias de assistências do paciente com ventilação mecânica ${ }^{10}$.

Sabe-se, que a sujidade na cavidade oral facilita a instalação de infecções oportunistas, onde deve ser realizada a completa higiene oral incluindo língua, dentes, palato, gengivas, etc. Observando o fluxo salivar e atentar para a realização da aspiração, evitando que o paciente broncoaspire, com a finalidade de prevenção de infecções".

TABELA 1 - Resultados da Pesquisa Importância da Higiene Oral com descritores em separados

\begin{tabular}{l|c|c|c}
\hline Descritores & Medline & Lilacs & BDENF \\
\hline Equipe de assistência em enfermagem & 53743 & 2355 & 1269 \\
\hline Unidade de terapia intensiva & 38014 & 3711 & 1238 \\
\hline Cuidados de enfermagem & 216641 & 11607 & 7060 \\
\hline
\end{tabular}

Fonte: Bases de dados, 2015.

TABELA 2 - Resultados da Pesquisa Importância da Higiene Oral com descritores combinados

\begin{tabular}{l|c|c|c}
\hline \multicolumn{1}{c|}{ Descritores } & Medline & Lilacs & BDENF \\
\hline Equipe de assistência em enfermagem /unidade de terapia intensiva & 1351 & 118 & 74 \\
\hline Equipe de assistência em enfermagem/cuidados de enfermagem & 3075 & 341 & 292 \\
\hline Unidade de terapia intensiva/cuidados de enfermagem & 3142 & 707 & 575 \\
\hline
\end{tabular}

Fonte: Bases de dados, 2015.

TABELA 3 - Resultados da Pesquisa Importância da Higiene Oral com descritores combinados

\begin{tabular}{|c|c|c|c|}
\hline Descritores & Medline & Lilacs & BDENF \\
\hline $\begin{array}{l}\text { Equipe de assistência em enfermagem/ unidade de } \\
\text { terapia intensiva/cuidados de enfermagem }\end{array}$ & 100 & 201 & 157 \\
\hline
\end{tabular}

Fonte: Bases de dados, 2015. 
TABELA 4 - Seleção dos Artigos da Pesquisa Importância da Higiene Oral

\begin{tabular}{|c|c|c|c|}
\hline ANO & AUTOR & TITULO & BASE \\
\hline 2006 & $\begin{array}{l}\text { Freire, I.L.S.; Farias, } \\
\text { G.M.; Ramos, C.S; }\end{array}$ & $\begin{array}{l}\text { Prevenindo pneumonia nosocomial: cuidados } \\
\text { da equipe de saúde ao paciente em ventilação } \\
\text { mecânica invasiva }\end{array}$ & BDENF \\
\hline 2006 & $\begin{array}{l}\text { Oliveira, A.C; Clemente, } \\
\text { W.T.; Lucas, T.C.; } \\
\text { Martinho, G.H.; }\end{array}$ & $\begin{array}{l}\text { Infecções hospitalares e resistência microbiana } \\
\text { em Unidade de Cuidados Intensivos de um } \\
\text { Hospital Universitário }\end{array}$ & BDENF \\
\hline 2008 & $\begin{array}{l}\text { Santos, P.S.S.; Mello, } \\
\text { W.R; Wakim, R.C.S.; } \\
\text { Paschoal, M.A.G.; }\end{array}$ & $\begin{array}{l}\text { Uso de solução bucal com sistema enzimático } \\
\text { em pacientes totalmente dependentes de } \\
\text { cuidados em unidade de terapia intensiva }\end{array}$ & LILACS \\
\hline 2009 & $\begin{array}{l}\text { Azambuja, P.M.; Neto, } \\
\text { E.C.; Santos, L.R.C.; } \\
\text { Knibel, M.F.; }\end{array}$ & $\begin{array}{l}\text { Pneumonia associada à ventilação mecânica: } \\
\text { epidemiologia e impacto na evolução clínica de } \\
\text { pacientes em uma unidade de terapia intensiva }\end{array}$ & LILACS \\
\hline 2010 & $\begin{array}{l}\text { Pombo, C.M.N.; Almeida, } \\
\text { P.C.; Rodrigues, J.L.N.; }\end{array}$ & $\begin{array}{l}\text { Conhecimento dos profissionais de saúde na } \\
\text { Unidade de Terapia Intensiva sobre prevenção } \\
\text { de pneumonia associada à ventilação }\end{array}$ & LILACS \\
\hline 2010 & $\begin{array}{l}\text { Kahn, S.; Mangialardo, } \\
\text { E.S.; Garcia, C.H.; } \\
\text { Namen, F.M.; Júnior, J.G.; } \\
\text { Machado, W.A.S; }\end{array}$ & $\begin{array}{l}\text { Controle de infecção oral em pacientes } \\
\text { internados: uma abordagem direcionada aos } \\
\text { médicos intensivistas e cardiologistas }\end{array}$ & LILACS \\
\hline 2011 & $\begin{array}{l}\text { Oliveira, T.F.L. ; Filho, } \\
\text { I.S.G.; Passos, J.S.; } \\
\text { Cruz, S.S.; Oliveira, } \\
\text { M.T.; Trindade, S.C.; } \\
\text { Machado, A.S.; Coelho, } \\
\text { J.M.F.; Santos, C.M.L.; } \\
\text { Cerqueira, E.M.M.; }\end{array}$ & $\begin{array}{l}\text { Fatores associados à pneumonia nosocomial } \\
\text { em indivíduos hospitalizados }\end{array}$ & LILACS \\
\hline 2012 & $\begin{array}{l}\text { Aguiar, A.S.C.;Mariano, } \\
\text { M.R.; Almeida, L.S.; } \\
\text { Cardoso, M.V.L.M.L.; } \\
\text { Pagliuca, L.M.F.; } \\
\text { Rebouças, C.B.A; }\end{array}$ & $\begin{array}{l}\text { Percepção do enfermeiro sobre promoção da } \\
\text { saúde na Unidade de Terapia Intensiva }\end{array}$ & LILACS \\
\hline 2012 & $\begin{array}{l}\text { Souza, A.F.; Guimarães, } \\
\text { A.C.; Ferreira, E.F.; }\end{array}$ & $\begin{array}{l}\text { Avaliação da implementação de novo protocolo } \\
\text { de higiene bucal em um centro de terapia } \\
\text { intensiva para prevenção de pneumonia } \\
\text { associada à ventilação mecânica. }\end{array}$ & LILACS \\
\hline 2013 & $\begin{array}{l}\text { Dereli, N.; Ozayar, E.; } \\
\text { Degerli, S.; Sahin, S.; } \\
\text { Koç, F.; }\end{array}$ & $\begin{array}{l}\text { Três anos de avaliação das taxas de infecção } \\
\text { nosocomial em UTI }\end{array}$ & LILACS \\
\hline
\end{tabular}

Fonte: Bases de dados, 2015.

O ambiente hospitalar é propicio de vários tipos de infecções cabe o profissional de saúde estabelecer maneiras de minimizar essas possíveis intercorrências. Uma das maneiras de reduzir o risco de infecção é promover ao paciente uma higienização oral diária e adequada, promovendo assim também o seu conforto e diminuindo sua estadia hospitalar'2.

É de vital importância promover a prevenção de patologias ligadas a precariedade da higiene oral.Tendo em vista que o enfermeiro tem a incumbência de realizá-la de forma eficaz, afim de evitar complicações que possam agravar o quadro clínico do paciente, podendo levá-lo a óbito. Impor- tante ressaltar que a cavidade oral sofre continua colonização bacteriana, e que concomitantemente a esse processo, ocorre uma limpeza natural, realizada através dos movimentos da mastigação de alimentos duros, da movimentação de língua e bochechas pela fala, que diminuem essa colonização. No entanto, ao se falar do paciente crítico, em uma unidade de terapia intensiva, nos deparamos com outra realidade, onde essa limpeza, não ocorre de maneira eficaz, devido a sedação e procedimentos invasivos. Sendo assim, com a impossibilidade dessa limpeza natural, devido a prótese ventilatória, algumas condutas podem evitar complicações e promover o bem-estar do paciente, como a higiene oral. 
O paciente sob utilização de ventilação mecânica, hospitalizado em unidade de terapia intensiva, fica impossibilitado de realizar o autocuidado. Além disso, com o uso do tubo orotraqueal a boca do paciente permanece aberta e o uso de sedativos propiciam a diminuição do fluxo salivar, ou seja, a xerostomia, acumulando bactérias que devem ser removidas mecanicamente pela escovação realizada pelo enfermeiro.

Cabe ao enfermeiro toda a assistência ao paciente com prótese ventilatória. É preciso estabelecer metas diárias de cuidados com a higiene oral, orientando toda a equipe e ressaltando a importância de evitar complicações e infecções e promover assim o bem-estar do paciente, que se encontra impossibilitado de realizar a sua própria higiene.

É fundamental que a equipe de enfermagem se conscientize que quanto maior e eficaz o cuidado ao paciente, menor será o seu tempo de permanência no ambiente hospitalar.

\section{Categoria 2 - Complicações decorrentes da falta de higiene oral.}

É de suma importância ter cuidados criteriosos ao manipular pacientes com dispositivos invasivos, pois os mesmos estão susceptíveis a adquirir infecções. É preciso usar a paramentação adequada, realizar técnicas estéreis, higienização das mãos a fim de reduzir o índice de infecção hospitalar ${ }^{13}$.

A pneumonia nosocomial, é uma das infecções mais desenvolvidas em pacientes com ventilação mecânica devido à falta de higienização oral onde facilita a colonização de bactérias. A negligência da higienização bucal é o principal fator de infecção no período de internação hospitalar ${ }^{14}$.

"A medicação tem ligação direta com a diminuição do fluxo salivar, ou seja, a xerostomia que somado com o fato do paciente estar com a boca aberta no tubo contribui para a proliferação de bactérias acarretando infecções”|'

"Quando o paciente é induzido a prótese ventilatória, seja por qual for a patologia, um dos fatores determinantes para a diminuição do tempo de internação é a higienização oral correta, pois através do mesmo prevenimos infecções"16.

Sendo a cavidade oral porta de entrada de microrganismos, a execução da higiene oral tem valor significativo, pois a realização deficiente ou ausência da mesma facilita a colonização de patógenos na cavidade oral, favorecendo a instalação de infecções, como por exemplo a pneumonia nosocomial.

Existe hoje no mercado diversos produtos para a higiene oral, cabe ao enfermeiro escolher o qual gera mais benefício para o paciente. É importante que o produto de escolha para a higiene oral seja o mais adequado, a fim de promover um ambiente oral "imune" às afecções, diminuindo o risco de complicações locais e sistêmicas.
O âmbito hospitalar é contaminado. Outros fatores agravantes são a baixa imunidade, os procedimentos invasivos, a utilização de medicações, o rebaixamento do nível de consciência, entre outros. Esses fatores podem aumentar o tempo de internação, levando a um ciclo vicioso: procedimentos invasivos, exposição a microrganismos e maior tempo de internação.

A principal via de instalação da pneumonia nosocomial é a cavidade oral, caracterizada pela colonização de bactérias que se proliferam devido à falta de higiene oral e tendo como agravante a xerostomia, ocorrendo a instalação nos pulmões.

\section{Categoria 3 - Educação em Enfermagem.}

"É importante em uma equipe multiprofissional incluir equipe de odontologia no cuidado ao paciente crítico, para um conjunto estabelecer diretrizes na assistência, a fim de reduzir possíveis complicações da falta ou a ineficiência da higiene oral" 17 .

É essencial a educação dos profissionais de saúde e da população acerca da importância da higiene oral. A população deve estar conscientizada que a higiene oral eficaz previne infecções cobrando assim os profissionais envolvidos na assistência a técnica correta quando seus familiares estiverem internados ${ }^{18}$.

"O conhecimento da equipe contribui, pois, a troca de conhecimento juntamente com a educação continuada são fundamentais para contribuir na prevenção de infecções que estão relacionadas ao conhecimento dos profissionais"'19.

A educação e o conhecimento são mecanismos que se obtém durante toda a vida profissional, é preciso entender a real necessidade de um problema e colocar em prática todo o seu conhecimento, garantindo o resultado imediato. É através de estudos, capacitações e aprimoramentos que se consegue estabelecer diretrizes em determinadas situações.

Toda equipe precisa ser informada sobre a importância dos cuidados diários ao paciente, que por mais simples que seja a assistência, no final se tem o resultado. Existem muitas patologias, e quando se dá conta, constata-se que por vezes, muitas delas poderiam ser evitadas se fosse estabelecido um plano de cuidado correto.

É fundamental estabelecer metas diárias de um plano de cuidado, criar e desenvolver planos de assistência e protocolos em conjunto com a equipe, buscando assim reduzir a estadia do paciente no ambiente hospitalar, com essas metas em prática também reduzimos as estatísticas de infecção.

A educação continuada é uma das atividades de promoção da saúde, realizada pelo enfermeiro com o intuito de prevenir doenças, diminui infecções e promover a humanização na assistência. 
O papel do enfermeiro na unidade de terapia intensiva deve ir além da realização de procedimentos invasivos e da atenção aos sinais e sintomas apresentados pelo paciente. $O$ enfermeiro deve prestar assistência ao indivíduo como todo, identificar problemas e riscos de complicações, orientar a equipe sobre a melhor forma de suprir as necessidades dos pacientes visando a eficácia na recuperação.

\section{CONSIDERAÇÕES FINAIS}

Ao se falar da atuação da equipe de enfermagem frente a realização da saúde oral dos pacientes internados, em unidades de terapia intensiva, evidenciou-se uma escassez de materiais, artigos científicos e protocolos que abordassem essa temática. Sendo a maioria dos estudos existentes, direcionados para a área odontológica.

No que se diz respeito ao empenho para realizar a pesquisa, pode-se afirmar que foram incessantes as buscas e observou-se a necessidade de publicações a serem divulgadas por enfermeiros a respeito de higiene oral como um cuidado em âmbito geral e não só em pacientes internados em unidade de terapia intensiva.

É muito limitado quaisquer tipos de informação ou esclarecimento no conceito da higiene oral em pacientes intubados. É visível a importância da higiene oral eficaz no dia a dia do paciente em uso de prótese ventilatória, pois a higiene oral promove conforto, evita complicações, quadros infecciosos e até mesmo reduz o tempo de permanência em ambiente hospitalar.

A precariedade da informação existe, porém, cabe ao enfermeiro orientar a sua equipe, pesquisar métodos e planos de cuidados, informar a família quanto a importância do procedimento e colocar todas as intervenções na rotina de enfermagem, garantindo assim, uma assistência completa e de valia.Para aperfeiçoar a prática de higiene oral, é de grande relevância implementar uma conduta adequada, através da utilização de protocolos assistenciais, destacando o valor das informações e os registros de enfermagem.

É notório que através do estudo realizado, podemos evidenciar o quanto a atuação do enfermeiro, vem a contribuir para a promoção da saúde de cliente críticos, minimizando desta forma, o índice de complicações relacionadas, a precariedade da realização correta da higiene oral.

A recomendação do estudo certifica o aperfeiçoamento do enfermeiro acerca da assistência de higiene oral ao paciente na terapia intensiva. É relevante a criação de programas, treinamentos e especializações nas unidades de terapia intensiva, visando a melhoria na assistência e crescimento profissional. 


\section{REFERÊNCIAS}

I. ARAÚJO, R.J.G.; OLIVEIRA, L.C.G.; HANNA, L.M.O.; CORRÊA, A.M.; CARVALHO, L.H.V.; ALVARES, N.C.F. Análise de percepções e ações de cuidados bucais realizados por equipes de enfermagem em unidades de tratamento intensivo. Revista Brasileira Terapia Intensiva, 2010 a.

2. POTTER P.A. M.; PERRY A.G. Fundamentos de enfermagem. Rio de Janeiro: Elsevier, 2009.

3. KNOBEL, E. Terapia Intensiva: Enfermagem. São Paulo: Editora Atheneu, 2010.

4. COFEN - CONSELHO FEDERAL DE ENFERMAGEM (BR). Lei n. 7498 de 1986: Dispõe sobre a regulamentação do exercício da enfermagem e de outras providências. Disponível em: http://www.cofen.gov.br/lei-n-749886-de-25de-junho-de-1986_416I.html.

5. ORLANDINI G. M.; LAZZARI C.M. Conhecimento da equipe de enfermagem sobre higiene oral em pacientes criticamente enfermos. Revista Gaúcha Enfermagem; 33(3): 34-4I, 2012.

6. SILVEIRA, I.R.; MAIA, F.O.M.; GNATTA, J.R.; LACERDA, R.A. Higiene Bucal: Prática relevante na prevenção da pneumonia hospitalar em pacientes em estado crítico. Acta Paul Enferm, 2010.

7. ARAÚJO, M.V.M.; VIEIRA, M.A.; BONAN, P.R.F.; COSTA, S.M. Atuação dos profissionais de enfermagem nos cuidados com a higiene bucal de idosos institucionalizados em Montes Claros- MG. Rev. APS, Juiz de Fora, v. I3, n. I, p. I017, jan./mar., 2010 b.

8. CERVO A.L.; BERVIAN P.A.; SILVA R. Metodologia Científica $6^{\mathrm{a}}$ Ed. São Paulo: Editora Pearson, 2006.

9. MASCARENHAS, S. A. Metodologia Científica. São Paulo: Editora Pearson, 2012.

I0. Freire, I.L.S.; Farias, G.M.; Ramos, C.S; Prevenindo pneumonia nosocomial: cuidados da equipe de saúde ao paciente em ventilação mecânica invasiva 2006
II. Santos, P.S.S.; Mello, W.R; Wakim, R.C.S.; Paschoal, M.A.G.; Uso de solução bucal com sistema enzimático em pacientes totalmente dependentes de cuidados em unidade de terapia intensiva 2008

12. KAHN, S.; GARCIA, C.H.; JÚNIOR,J.G.; NAMEN, F.M.; MACHADO, W.A.S.; JÚNIOR, J.A.S.; SARDENBERG, E.M.S.; EGREJA, A.M. Avaliação da existência de controle de infecção oral nos pacientes internados em hospitais do estado do Rio de Janeiro. Ciência e saúde coletiva, 13(6): 1825I83I, 2008.

13. Dereli, N.; Ozayar, E.; Degerli, S.; Sahin, S.; Koç, F.; Três anos de avaliação das taxas de infecção nosocomial em UTI 2013

I4. Oliveira, T.F.L. ; Filho, I.S.G.; Passos, J.S.; Cruz, S.S.; Oliveira, M.T.; Trindade, S.C.; Machado, A.S.; Coelho, J.M.F.; Santos, C.M.L.; Cerqueira, E.M.M.; Fatores associados à pneumonia nosocomial em indivíduos hospitalizados20II

15. Oliveira, A.C; Clemente, W.T.; Lucas, T.C.; Martinho, G.H.; Infecções hospitalares e resistência microbiana em Unidade de Cuidados Intensivos de um Hospital Universitário 2006

16. Rodrigues Y.C.S.; Studart R.M.B.; Andrade I.R.C.; Citó M.C.O.; Melo E.M.; Barbosa I.V.Ventilação Mecânica: evidências para o cuidado de enfermagem. Ceará, 2012.

17. Souza, A.F;; Guimarães, A.C.; Ferreira, E.F;; Avaliação da implementação de novo protocolo de higiene bucal em um centro de terapia intensiva para prevenção de pneumonia associada à ventilação mecânica.20I2

18. Aguiar, A.S.C.;Mariano, M.R.; Almeida, L.S.; Cardoso, M. V.L.M.L.; Pagliuca, L.M.F.; Rebouças, C.B.A; Percepção do enfermeiro sobre promoção da saúde na Unidade de Terapia Intensiva 2012

19. Pombo, C.M.N.; Almeida, P.C.; Rodrigues, J.L.N.; Conhecimento dos profissionais de saúde na Unidade de Terapia Intensiva sobre prevenção de pneumonia associada à ventilação 2010 . 